\title{
Die Schenkelhalsfraktur (SHF) beim Erwachsenen
}

\author{
Konservative und kopferhaltende Therapie
}

Daniel Geissmann, Reto Babst

\section{Zusammenfassung}

Die Behandlung der medialen Schenkelhalsfraktur wird immer noch kontrovers diskutiert. Die zur Verfügung stehenden Klassifikationen sind in ihrer Wertigkeit limitiert, weshalb für den klinischen Alltag eine Unterscheidung in nicht dislozierte und dislozierte Schenkelhalsfrakturen genügend scheint. Nicht nur das chronologische Alter und der Frakturtyp sollten den Therapieentscheid für ein konservatives oder operatives Vorgehen beeinflussen, sondern Faktoren wie Aktivi- tätsgrad, Knochendichte und Comorbiditäten des Patienten. Akzeptierte Altersgruppen für eine kopferhaltende Therapie sind Patienten $<65$ Jahren. Für die Patientengruppe der $>65$ Jährigen scheint ein individualisiertes Vorgehen, gestützt auf die genannten Faktoren, mit einer relevanten Reduktion von Sekundäreingriffen assoziiert zu sein. Als operative, den Schenkelhals erhaltende Versorgung kommen in erster Linie die Verschraubung mit 3 kanülierten Schrauben und die Osteosynthese mit einer DHS infrage.

\section{Einleitung}

Die Behandlung der Schenkelhalsfraktur (SHF) wird auch heute noch, trotz einer über 100-jährigen Erfahrung in der operativen Behandlung dieser Frakturen, trotz über 2000 Publikationen und trotz Zunahme ihrer Inzidenz immer noch kontrovers diskutiert.

Der Chirurg steht deshalb vor dem Dilemma, den Hüftkopf zu erhalten oder ihn durch ein Kunstgelenk zu ersetzen. Die Kopferhaltung gibt meist die Gewähr einer guten Funktion, jedoch zum Preis einer eventuell höheren Rate an Sekundärkomplikationen, wie Sekundärdislokation, wie Nonunion und wie avaskuläre Kopfnekrose. Beim letzteren besteht das Risiko einer schlechteren Funktion durch einen Gelenkersatz, jedoch zum Preis fehlender Frakturheilungsstörungen.

Trotz einer Metaanalyse [13] und unzähligen retrospektiven Fallkontrollstudien fehlt bislang eine klare Evidenz für das

OP-JOURNAL 2002; 17: 106-112

(c) Georg Thieme Verlag Stuttgart · New York therapeutische Vorgehen bei kopfverhaltender Zielsetzung. Es erstaunt deshalb nicht, dass sich in der Behandlung dieser Frakturen ein sehr individualisierter Ansatz, welcher sich an verschiedenen Faktoren, wie Alter, Aktivitätsgrad, Knochenqualität, Frakturtyp und Gesundheitszustand des Patienten orientiert, allmählich durchsetzt.

\section{Epidemiologie}

SHF erleiden am häufigsten Individuen nach dem 60. Altersjahr. Das Durchschnittsalter dieser Patienten beträgt in den meisten Arbeiten zwischen 75 und 82 Jahren. Frauen sind davon häufiger betroffen. Das Verhältnis Frauen zu Männer beträgt in Abhängigkeit der beobachteten Population etwa 3-3,5: 1. Höheres Lebensalter und die hormonell bedingte, stärkere Osteoporose und geringere Muskelkraft sind diskutierte Faktoren für dieses ungleiche Verhältnis. Mangelernährung, verminderte körperliche Aktivität, schlechteres Sehvermögen, neurologische Defizite, ein schlechteres Gleichgewicht, veränderte Reflexe und eine Muskelatrophie sind weitere Faktoren, welche für das Entstehen solcher Frakturen nach einfachem Sturz aus dem Stand ver- antwortlich sind. In dieser Altersgruppe resultieren aus solchen Stürzen mehrheitlich mediale SHF. Intermediäre und laterale Schenkelhalsfrakturen dagegen sind wesentlich seltener. Sie betreffen eher jüngere Altersgruppen und treten als Folge von Hochgeschwindigkeitstraumen, wie Sturz vom Fahrrad auf die Hüfte, beim Ski- oder bei Skateboardfahren auf.

In Deutschland beträgt die Inzidenz der Schenkelhalsfrakturen für die Gesamtbevölkerung ca. 50/100000 Einwohner. Für die Bevölkerung der über 65-Jährigen steigt sie auf ca. 350/100000 Einwohner und bei den über 80 -Jährigen beträgt sie bereits etwa 600-900/100000 Einwohner. Die Tendenz ist steigend, wobei ein jährlicher Zuwachs von 3-5\% beobachtet wird [4].

\section{Gefäßversorgung des Schenkelhalses und des Femurkopfes}

Ein prognoserelevanter Faktor für die kopferhaltende Therapie ist zumindest eine partielle Integrität der Gefäßversorgung von Schenkelhals und Femurkopf. Die arterielle Femurkopfdurchblutung wird durch drei Gefäße gewährleistet. Den größten Anteil an der Femurkopfdurchblutung tragen die intrakapsulären Retinakulagefäße bei. Sie werden aus dem extrakapsulären Ring, welcher von der A. circumflexa femoris lateralis und medialis gebildet wird, gespeist (Abb.1). Für die Kopfdurchblutung weniger relevant sind die Arterie im Lig. capitis femoris (max. 30\% der Kopfperfusion) und die Gefäße des Knochenmarkkanals.

Die Gefäßarchitektur kann durch eine einwirkende Gewalt zerrissen oder die Kopfperfusion durch die intraartikuläre Druckerhöhung durch das Frakturhämatom kompromitiert werden. Eine Revaskularisation scheint jedoch über die Arterie des Lig. capitis femoris oder aber über den Frakturspalt möglich. Aus diesen Gründen erhält der Zeitpunkt der opera- 


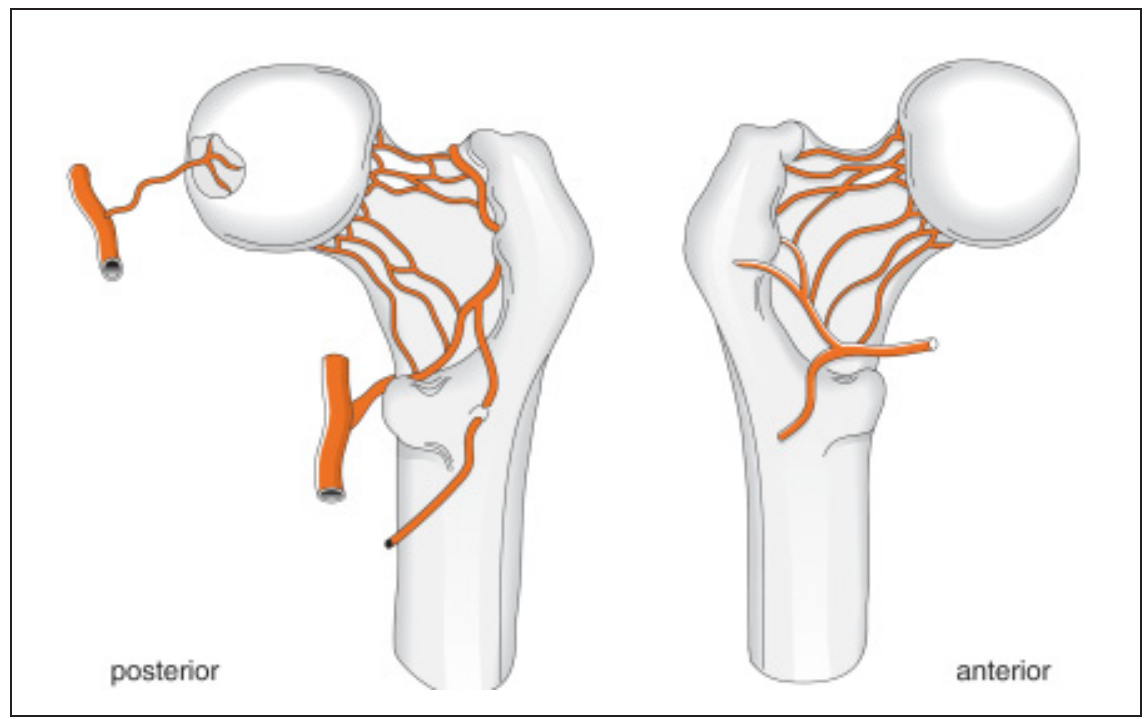

Abb.1 Blutversorgung des Schenkelhalses.

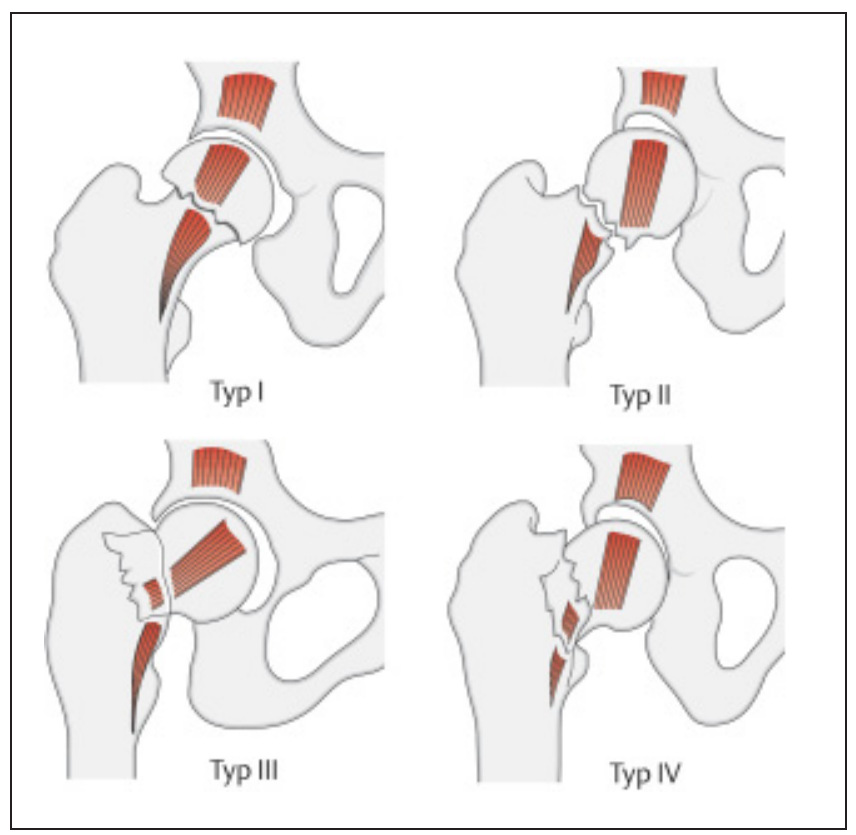

Abb. 2 Einteilung der SHF nach Garden in Typ I bis IV. Nach: (Robinson CM, Alho A, Court-Brown CM. (Eds). Femur. Arnold London 2002). dem sehr selten. Die für die Prognose einer Femurkopfnekrose angeblich relevante Unterscheidung der dislozierten Frakturen in eine teilweise (Garden Typ III) und eine komplett dislozierte Fraktur konnte in einer von Garden selbst publizierten Arbeit mit 1504 SHF [3] nicht reproduziert werden.

Die Klassifikation von Pauwels (Abb.3), welche einen prädiktiven Wert bezüglich der Entstehung einer Pseudarthrose besitzt, erlaubt oft erst intraoperativ eine adäquate Frakturklassifizierung, da der Frakturlinienverlauf meist erst intraoperativ unter Traktion und Innenrotation evident wird.

Ähnliches gilt auch für die AO-Klassifikation, welche Elemente beider vorgenannter Klassifikationen enthält und durch ihre zusätzliche Unterklassen für den klinischen Alltag zu komplex ist.

Für die tägliche Entscheidungsfindung scheint die Einteilung in dislozierte (nicht impaktierte) und undislozierte (impaktierte) Frakturen, wie sie Colles [6] bereits im letzten Jahrhundert beschrieben hat, genügend.

\section{Undislozierte (impaktierte) SHF}

Die Entscheidung zum konservativen Vorgehen orientiert sich am Risiko der Sekundärdislokation der undislozierten (impaktierten) Fraktur. Unzählige Faktoren, die eine Sekundärdislokation begünstigen, wurden in den letzten Jahren anhand retrospektiver Arbeiten postuliert. Aufgrund biomechanischer Überlegungen sind eine Retroversion von mehr als $20^{\circ}$, eine Valgusfehlstellung von mehr als $20^{\circ}$, eine Varusfehlstellung oder ein Pauwelswinkel von mehr als 45 Grad nachvollziehbare Gründe für ein erhöhtes Risiko zur Sekundärdislokation. In der Literatur fehlt dafür aber die Evidenz [16].

linien. Bei dislozierten SHF sind die relevanten Frakturlinien des Schenkelhalses oft nur schlecht beurteilbar. Das erklärt, warum die klinisch am häufigsten verwendete Garden Klassifikation (Abb.2) eine sehr schlechte Reproduzierbarkeit aufweist [7]. Die Unterscheidung der valgusimpaktierten Garden Typ I SHF, von der nicht impaktierten medialen SHF (Garden Typ II) ist ohne die Anfertigung einer 2. Ebene im Röntgenbild nicht möglich. Da auch im ap Strahlengang anscheinend nicht dislozierte Frakturen oft eine dorsale Impaktion aufweisen, ist eine Fraktur Garden Typ II anhand eines a.p. Bildes allein nicht bestimmbar und $\mathrm{zu}$ -
Während die angelsächsische Literatur die prophylaktische Verschraubung aller undislozierten Frakturen mit dem Argument einer 20\%-Rate an Sekundärdislokationen empfiehlt [18] lehnt die Gruppe um Raymakers et al. [16] dieses Vorgehen mit dem Argument ab, dass in $70 \%$ eine unnötige Intervention stattfindet. In einem Patientenkollektiv von 314 undislozierten SHF, welche funktionell behandelt wurden, kam es in 30\% zur Sekundärdislokation. Letztere wurde in $42 \%$ bei Patienten $>70$ Jahren und lediglich in $10 \%$ bei der Altersgruppe $<70$ Jahren 


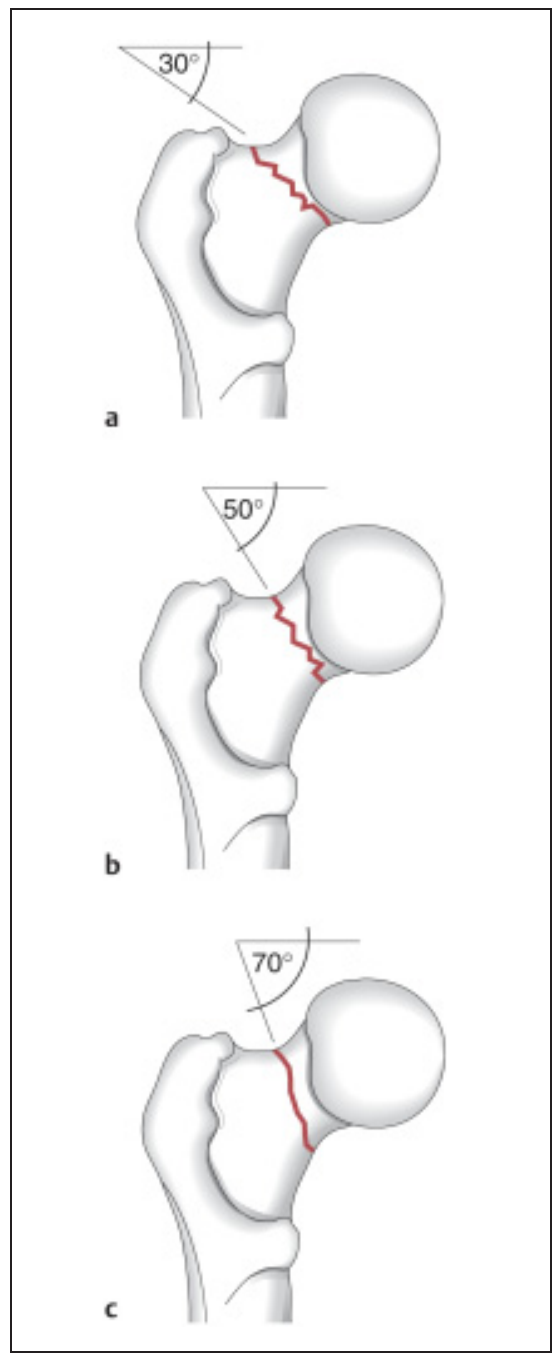

Abb.3 Einteilung der SHF nach Pauwels: A =Typ 1, B = Typ II, C= Typ III. (Nach: Chapman MW. Chapman's Orthopaedic Surgery. Lippincott Philadelphia 2002). beobachtet. Als Risikofaktoren wurden in einer Regressionsanalyse Comorbiditäten und das Alter $>70$ Jahre angegeben.

Die Wahrscheinlichkeit einer Sekundärdislokation bei einem gesunden Patienten $<70$ Jahre wurde auf $5 \%$, während sie für Patienten $>70$ Jahre mit mehreren Nebendiagnosen auf $79 \%$ berechnet wurden.

Diese Daten unterstützen ein individualisiertes Vorgehen, welches sich neben dem Frakturtyp an Faktoren wie biologisches Alter und Comorbidität orientiert.

Die Resultate der konservativen Behandlung undislozierter SHF (Abb. 4, 5) zeigen wahrscheinlich wegen fehlender Diskriminierung verschiedener Cofaktoren eine enorme Streuung zwischen 15$60 \%$. Kopfnekrosen nach konservativer und Therapie wurden in etwa $7-16 \%$ der Fälle beobachtet [16].

Im eigenen Vorgehen werden undislozierte impaktierte SHF beim alten polymorbiden Patienten, welche im axialen Bild keine Dislokation über $20^{\circ}$ aufweisen, funktionell behandelt. Bedingung sind unter anderem fehlende Schmerzen und die sofortige Mobilisation unter Vollbelastung. Beim Patienten unter 65 Jahren, kommt ein konservatives Vorgehen dann zur Anwendung, wenn im axialen Bild keine Retroversion und gute Knochensubstanz bestehen. Dies bietet eine gewisse Gewähr, dass relevante Verkürzungen und ein sekundäres Impingement bei der Hüftflexion, bedingt durch die Re- troversion des Femurkopfes, vermieden werden können. Eine gute Patientenkompliance scheint ebenfalls wichtig.

\section{Dislozierte SHF}

Während beim Patienten $<65$ Jahren mit normaler Knochenstruktur die kopferhaltende Therapie unbestritten ist, bestimmen bei Patienten in der Altersgruppe $>65$ Jahre neben der Fraktur zusätzliche Faktoren, wie die Knochenstruktur, die körperliche Aktivität und fehlende Comorbiditäten den Therapieentscheid zum Kopferhalt mittels einer Osteosynthese.

Der Singh-Index ist zur Beurteilung der Knochenstruktur aufgrund der schlechten Reproduzierbarkeit umstritten. Die Beurteilung der Knochendichte mit Dexa-Scan könnte diejenigen Patienten selektionieren, deren Knochenstruktur genügend Stabilität für eine Osteosynthese gewährleistet. Das Aktivitätsniveau des Patienten kann anhand des physiologischen Status-Score von Robinson et al. [17] bestimmt werden. Unter Anwendung dieses Scores bei der Patientengruppe $>65$ Jahre bis 84 Jahre konnten in der Gruppe mit Kopferhalt durch Osteosynthese, Reoperationen auf $<2 \%$ reduziert werden. Dies ist bei Betrachtung einer Reinterventionsrate von 35\% in der Metaanalyse von Lu-Yao et al. [13] bemerkenswert.

Unabhängig vom Alter und den erwähnten Faktoren wird beim Patienten mit einer Coxarthrose, einer pathologischen Fraktur oder bei Zusatzerkrankungen
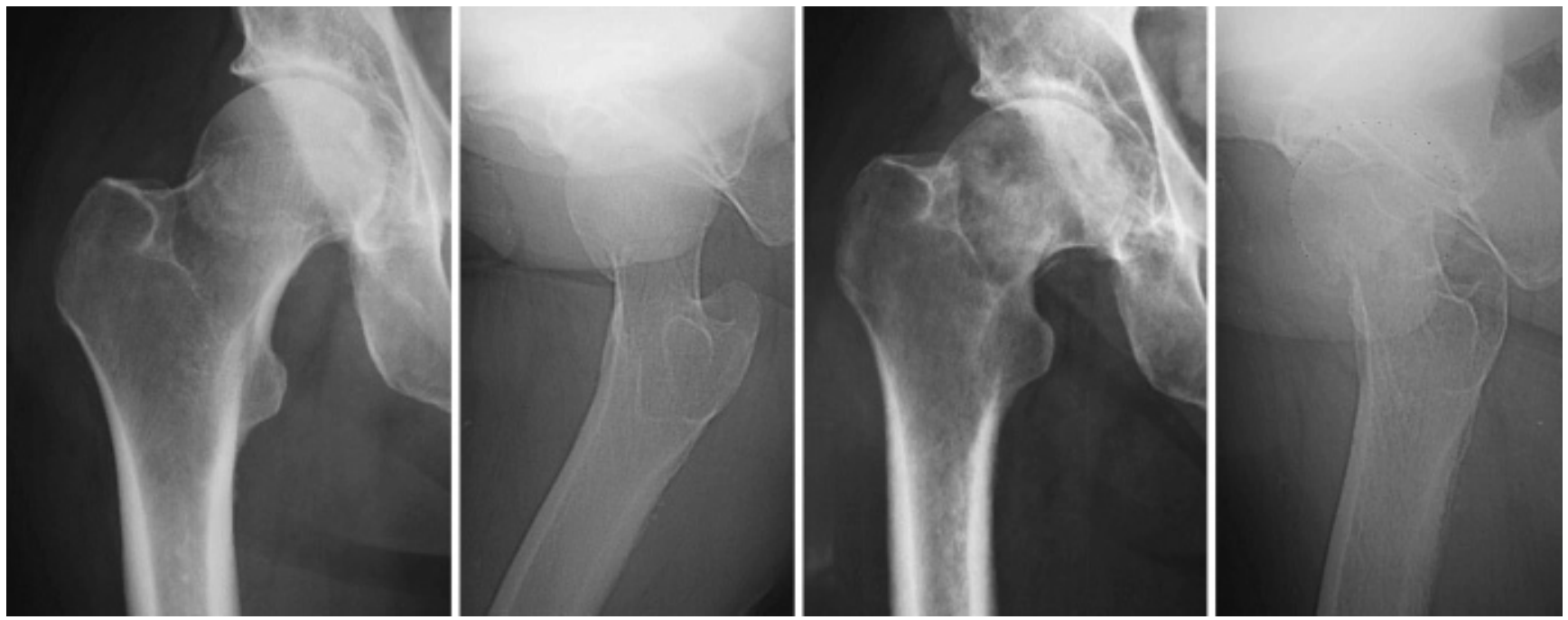

Abb. 4 W.J., 59j, AO-Typ 31 B1, Garden-Typ-1-Bilder links: rechtes Hüftgelenk a.p./axial mit einer Abduktionsfraktur (Garden Typ I) des Schenkelhalses, konservativ behandelt. Bilder rechts: Röntgenbilder nach 6 Monaten mit Osteolysen im Kopfbereich und Strukturvermehrung bei Verkürzung im Frakturbereich. 


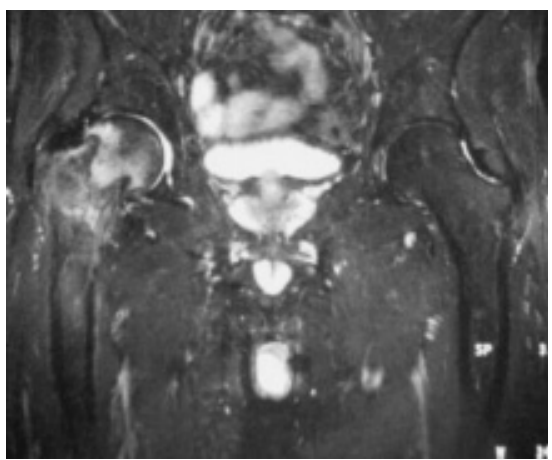

Abb. 5 W.J., 59j, AO-Typ 31 B1, Garden-Typ-IMRI-Befund des Beckens 6 Monate nach konservativ behandelter Abduktionsfraktur. Ödem im Frakturbereich des rechten Schenkelhalses als Zeichen einer Non union.

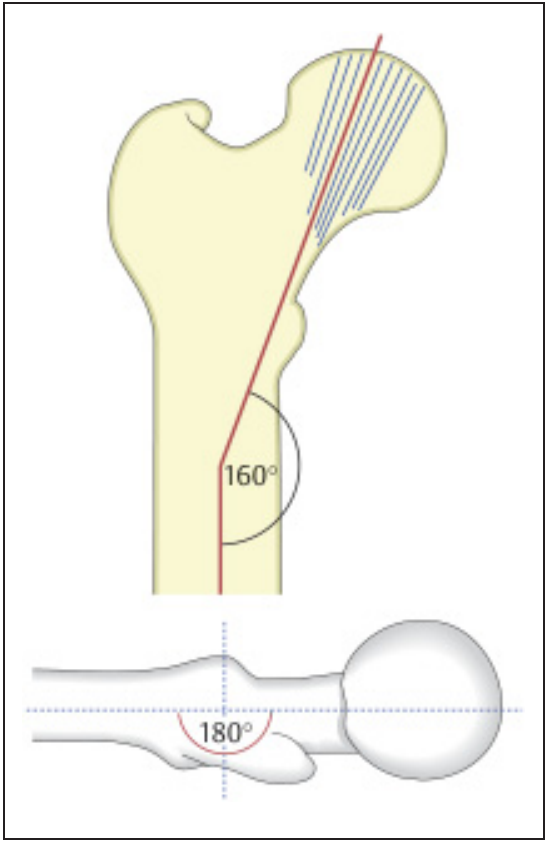

Abb. 6 Gardens Alignement-Index

wie einer PCP vom Kopferhalt Abstand genommen.

\section{Operationszeitpunkt}

Obwohl vereinzelte Autoren einen $\mathrm{Zu}-$ sammenhang zwischen einer Operation innerhalb von $6 \mathrm{~h}$ nach dem Unfall und einer Reduktion der Kopfnekroserate vermuten, wird ein solcher Zusammenhang von anderen Autoren nicht bestätigt. Bonnaire konnte eine kontinuierliche intraartikuläre Druckzunahme in den ersten $6 \mathrm{~h}$ bis auf $22 \mathrm{~mm} \mathrm{Hg}$ und in den nächsten 2 Tagen bis auf $48 \mathrm{~mm} \mathrm{Hg}$ zeigen. $\mathrm{Zu}$ dem wurde wiederholt der Zusammenhang der intraartikulären Druckerhöhung durch Extension und Innenrotation der betroffenen Extremität aufgezeigt [5].
Unter der Annahme, dass eine Gelenkkapselzerreißung nur in 5-15\% der Fälle stattfindet und damit die intraartikuläre Druckerhöhung als potentielle Ursache der Kopfischämie in der Mehrheit der Fälle nicht wegfällt, sollte bei allen SHF die einer kopferhaltenden Therapie zugeführt werden, eine rasche operative Intervention erfolgen. Auch wenn zunehmend Infrastrukturressourcen knapp sind, sollten diese Frakturen als dringliche Notfälle behandelt werden. Die zitierten pathophysiologischen Zusammenhänge lassen vermuten, dass damit zumindest ein Ischämiefaktor beseitigt werden kann. Die Kopfischämie bedingt durch Gefäßzerreißung und durch Kopfdislokation oder Impaktion ist dagegen nicht beeinflussbar.

\section{Reposition}

Das Ziel der Osteosynthese von SHF ist die möglichst gute Wiederherstellung der Anatomie und die stabile Fixation zur Verhinderung einer Pseudoarthrose, einer Kopfnekrose oder einer Sekundärdislokation. Zur intraoperativen Beurteilung der Reposition eignet sich der Garden-Alignement-Index (Abb.6), welcher sich an der Richtung der Knochentrabekel im a.p. und axialen Strahlengang orientiert.

Eine Reposition mit einem Garden Alignement-Index von $160-180^{\circ}$ im a.p. Bild und von $160-180^{\circ}$ im axialen Bild sollte angestrebt werden. Eine Valgusimpaktion bis $20^{\circ}$ wird dabei akzeptiert, jedoch nicht eine Varusstellung des Kopffragmentes.

Die Reposition wird am einfachsten mit Hilfe des Extensionstisches erreicht (Abb.6). Durch Extension und leichte Innenrotation ist die Retroversion des Femurkopfes oft korrigierbar. Bei der Reposition ohne Extensionstisch wird bei $90^{\circ}$ Hüftflexion der Oberschenkel nach innen rotiert und letzterer unter Zug in Streckstellung und in Abduktion gebracht [12]. Gelingt geschlossen keine genügende Reposition, so sollte zur offenen Reposition gewechselt werden. Damit wird ein Zusatzschaden durch forcierte Repositionsmanöver verhindert. Zudem kann bei der offenen Reposition die Durchblutung des Femurkopfes durch Anbohren oder durch Laserdopplerflussmessung überprüft werden [9]. Dies erlaubt eine zuverlässige Angabe bezüglich einer Kopfnekrose.

In Bezug auf die Repositionsgenauigkeit wurde zwischen geschlossener und offe- ner Osteosynthese großer Serien kein Unterschied gefunden $[3,16]$.

Die Inzision der Gelenkkapsel zur Entlastung des Kapselhämatoms sollte aus pathophysiologischen Gründen zur Verbesserung der Kopfdurchblutung bei allen Patienten im Rahmen der Osteosynthese auch beim geschlossenen Verfahren erfolgen.

\section{Osteosynthese}

Am häufigsten werden mediale SHF mit Spongiosaschrauben stabilisiert. Der Patient ist in Rückenlage auf dem Extensionstisch und der Zugang erfolgt ca. $3 \mathrm{~cm}$ unterhalb des Trochanter majors. Durch den Vastus lateralis hindurch wird das proximale Femur zwischen Hohmannhacken dargestellt. Beim perkutanen Vorgehen wird die Höhe der Stichinzisionen durch das Auflegen eines Führungsdrahtes auf die Haut in der Region des Schenkelhalses mit Hilfe des Bildverstärkers bestimmt.

Im eigenen Vorgehen werden kanülierte, selbstbohrende und selbstschneidende Schrauben der Dimension 7,3 mm mit Gewindelängen 16 oder $32 \mathrm{~mm}$ verwendet. Der Vorteil kanülierter Schrauben ist das korrekte Platzieren der Führungsdrähte ohne relevanten Knochensubstanzverlust, bevor die definitiven Spongiosazugschrauben eingebracht werden (Abb.8). Alle Schrauben sollten in den zentralen $2 / 3$ des Femurkopfes positioniert sein. Um dies zu erreichen, sollte die erste Schraube entlang des Calcar im a.p. Bild und in der Mitte im axialen Bild platziert werden. Die 2. Schraube sollte dorsal oberhalb der ersten Schraube im a.p. Bild und dorsal im hinteren Drittel des Schenkelhalses im axialen Bild positioniert sein. Die dritte Schraube sollte im a.p. Bild über den beiden bereits eingebrachten Schrauben und im axialen Bild im mittleren bis vorderen Drittel des Schenkelhalses $\mathrm{zu}$ liegen kommen (Abb.9). Wichtig ist es, den Eintritt der kaudalsten Schraube nicht unterhalb der Trochanter minor Ebene einzubringen, oder die zwei kaudalen Schrauben auf der gleichen Höhe einzubringen, da sonst die Gefahr einer Sekundärfraktur nach Aufnahme der Belastung besteht [18]. Einzelne Autoren verwenden auch nur zwei, andere jedoch bis zu 4 Schrauben. Letztere sollen gemäß In vitro Studien vor allem bei starker posteriorer Trümmerzone signifikant stabiler sein als 3 Schrauben [11]. Vor allem in skandinavischen Ländern werden anstelle der 

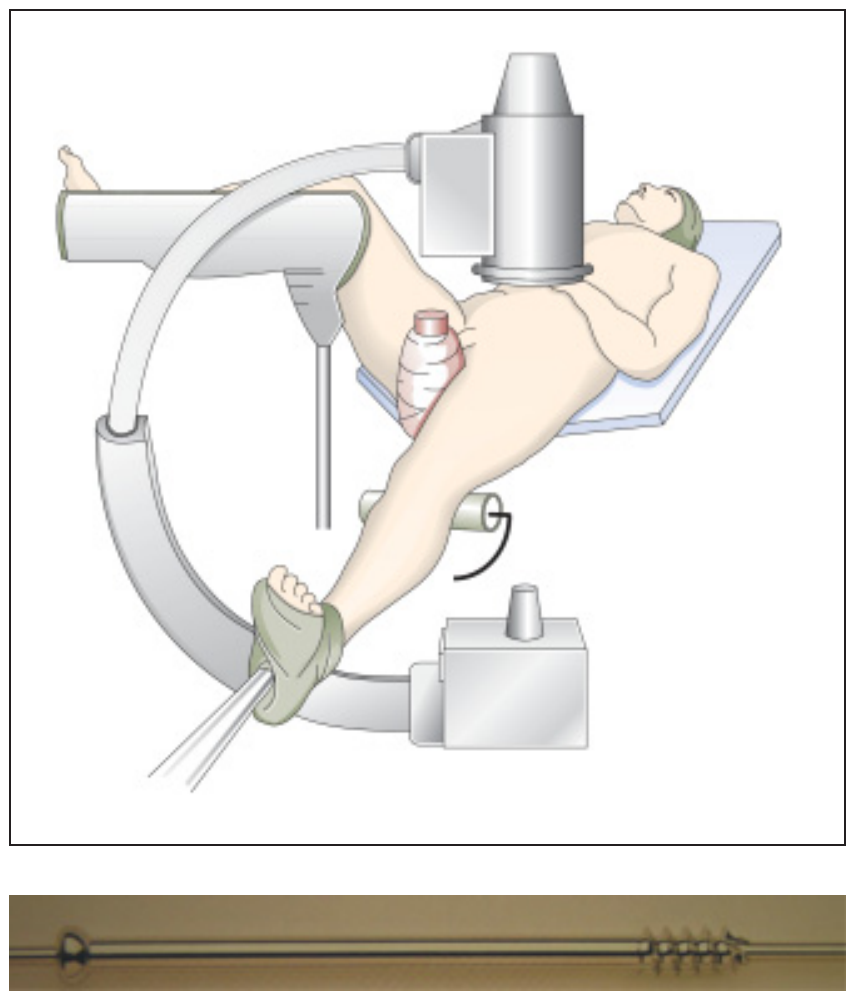

Abb. 8 Spongiosazugschraube mit dem noch liegenden Führungsdraht.

kanülierten Schrauben oft UppsalaSchrauben, Hansson-Stifte oder Ulleval Schrauben verwendet.

All diesen Implantaten gemeinsam ist, dass sie parallel zueinander und zur Schenkelhalsachse eingebracht werden. Dies erlaubt ein Gleiten der Schrauben und ein kontrolliertes Zusammensintern der Fraktur unter der Belastung.
Abb. 7 Lagerung auf dem Extentionstisch bei dem SHF.
Schlechte Knochenqualität, eine Schraubenpositionierung in der apikalen Femurkopfhemisphäre und eine Varusreposition sind assoziiert mit einem Ausbrechen von Schrauben aus dem Femurkopf. Die Angaben über die Inzidenz einer Nonunion variieren in der Literatur sehr stark und streuen zwischen 2-30\%. Neben einer vertikalen Fraktur (Pauwels III), sind die Varusreposition, das Patienten- alter und die Tatsache der Dislokation gute Prädiktoren für eine Nonunion (Pseudoarthrose). Die Rate an avaskulären Kopfnekrosen beträgt nach dislozierten SHF zwischen 10 und 20\% [18]. Das Kopfnekroserisiko soll, gemessen an einer retrospektiven Datenerhebung mit dem Ausmaß der Dislokation und dem Zeitpunkt der Operation, korrelieren [14].

Die DHS mit oder ohne Antirotationsschraube (Abb.10) wird von einzelnen Autoren für alle SHF verwendet, während viele dieses Implantat nur für spezielle Frakturtypen reservieren. Obwohl Parker [15] in einer Metaanalyse keinen Unterschied zwischen beiden Implantaten in Bezug auf die klinischen Resultate fand, war die Pseudoarthroserate nach DHS bei Ansis S et al. [1] mit $1 \%$ und bei Fuglesang et al. [8] mit 8\% sehr gering. Die Kopfnekroserate war jedoch in dieser Serie $37 \%$, was eher gegen eine generelle Anwendung dieses Implantats spricht. Nach Kadaverstudien von Baitner [2] ist die Stabilität der DHS im Vergleich zu Schrauben bei sagittal verlaufenden Frakturen des Typs Pauwels III besser. Die DHS wird deshalb vor allem für diesen Frakturtyp erfolgreich angewandt.

Andere Implantate wie die Klingenplatte finden für die Primärversorgung dieser Frakturen aufgrund der Gefahr von Klingenprotrusionen nur mehr selten Anwendung. Dieses Implantat hat jedoch seinen Stellenwert in der Behandlung von Pseudoarthrosen nach SHF behalten.
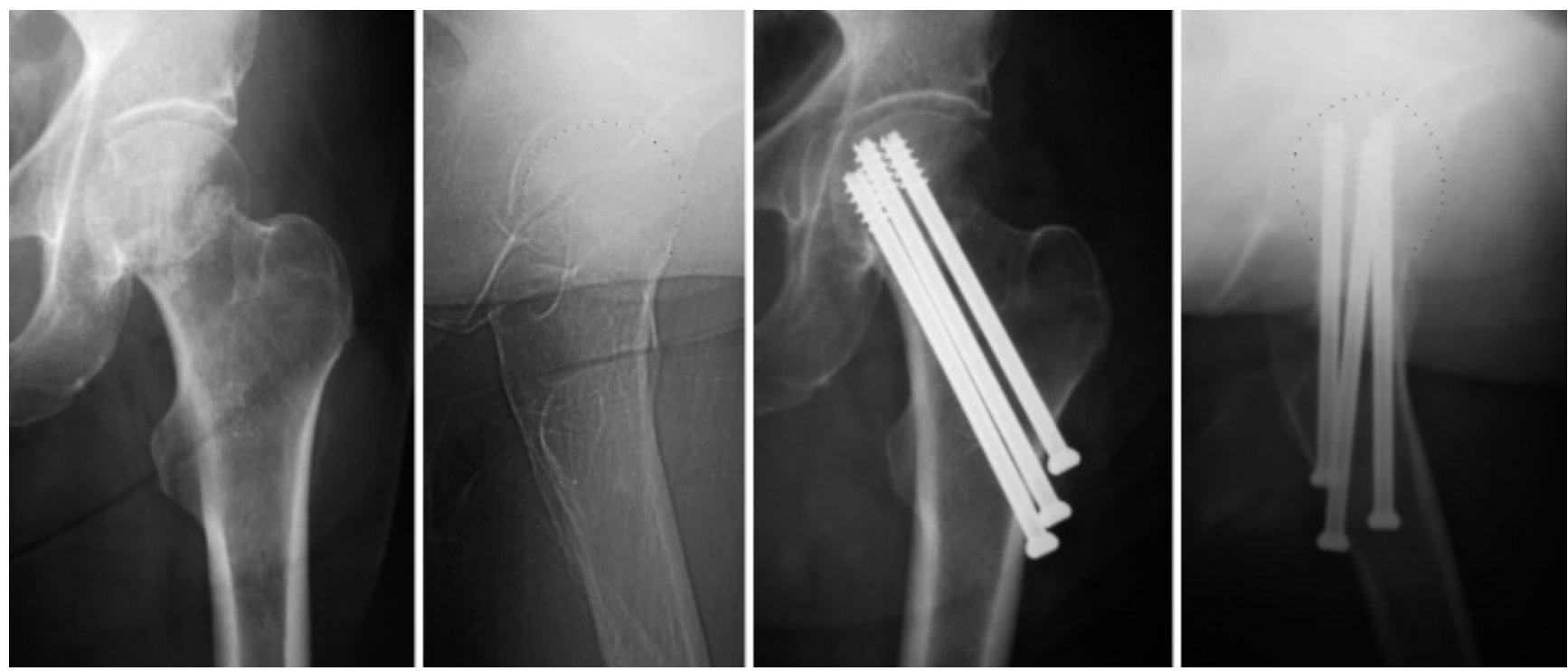

Abb.9 S.R., 63j., AO-Typ B 1, Garden-Typ I Bilder links: Hüfte links a.p./axial mit einer Abduktionsfraktur des Schenkelhalses. Bilder rechts: 6 Wochen nach Verschraubung mit 3 kanülierten 7,3-mm-Spongiosazugschrauben. Die Retroversion ist aufgehoben. 

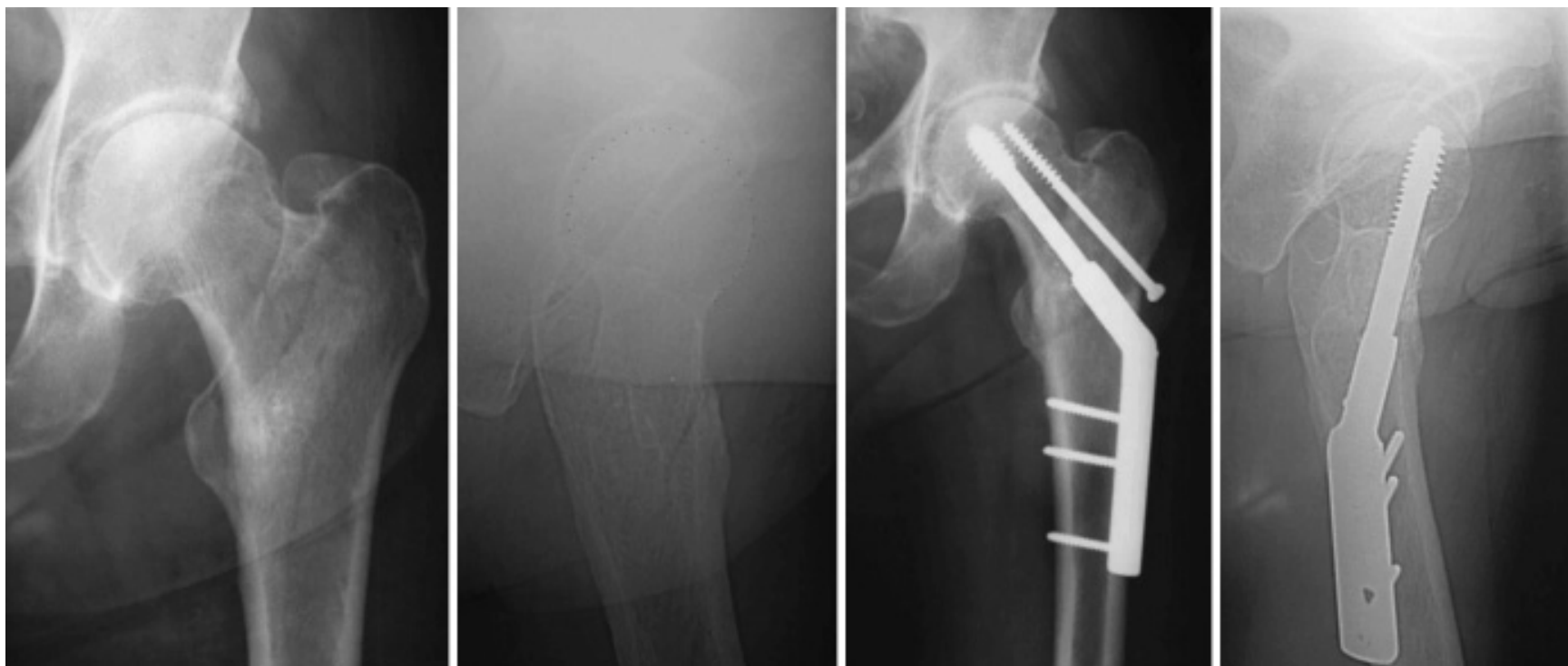

Abb.10 B. J., 89j., AO-Typ B 2, Pauwels-Typ III links: Röntgenbild des linken Hüftgelenks a.p./axial: laterale, praktisch undislozierte Schenkelhalsfraktur. Rechts: 6 Wochen nach Versorgung mit DHS (kann auch nur 2-Loch-Platte sein) und Antirotationsschraube.

\section{Nachbehandlung}

Konservativ und operativ behandelte SHF können aufgrund vieler Erfahrungsberichte ohne wesentliche Folgeprobleme voll belastet werden.

Die Art der operativen Versorgung scheint dabei keine Rolle zu spielen. Instrumentierte Hüftprothesen, welche die Druckbelastung im Femurkopf erfassten, konnten zeigen, dass zwischen der Belastung beim Gehen und dem isometrischen Strecken des Beines im Bett lediglich ein geringer Belastungsunterschied besteht.

\section{Tipps und Tricks}

Eine anatomienahe Frakturreposition mit korrekter Schraubenplatzierung ist der Schlüssel zum Erfolg einer kopferhaltenden Therapie bei SHF. Die Osteosynthese sollte bei dislozierten Frakturen erst nach erfolgter Reposition durchgeführt werden. Die Auflage eines Führungsdrahtes auf die ventrale Fläche des Schenkelhalses erleichtert die Positionierung der Kirschner-Drähte in Bezug auf die Antetorsion. Eine leichte Retroversion des Kopf-/Halsfragmentes nach dem Repositionsmanöver auf dem Extensionstisch kann gelegentlich durch einen GewindeKirschner-Draht, welcher in den dorsalen Anteil des Kopf/Halsfragmentes eingebracht wird, durch ein „Wegstoßen des Fragmentes" beim Einbringen, aufgehoben werden.
Eine Möglichkeit die Stabilität der Osteosynthese zu erhöhen ergibt sich durch das Einbringen von flüssigem Calciumphosphat Zement im Bereich der Trümmerzone. Erste klinische Erfahrungen mit dieser lokalen Augmentation sind günstig [10].

Die intraoperative Beobachtung der Trabekellinien, wie sie der Alignement-Index nach Gardner beschreibt, kann als Orientierungshilfe für eine korrekte Reposition dienen. Sollte die Reposition geschlossen nicht optimal möglich sein, so muss diese offen erfolgen oder aber ein prothetischer Ersatz vorgenommen werden.

Insbesondere beim jüngeren Patienten sollten Schrauben erst nach vorherigem Gewindeschneiden eingebracht werden. Dies gilt vor allem für die DHS, bei der durch das Einbringen der Schrauben eine relevante Rotationskraft erzeugt werden kann. Deshalb ist es bei Verwendung dieses Implantats wichtig, die Rotationskräfte durch vorgängig eingebrachte Kirschner-Drähte zu neutralisieren.

\section{Schlussfolgerung}

Die Versorgung der SHF stellt auch in $\mathrm{Zu}-$ kunft eine große Herausforderung für den Chirurgen dar. Zum Einen wird diese Fraktur aufgrund der demografischen Entwicklung in den nächsten Jahrzehnten zunehmen und zum Anderen werden uns die stets steigenden Gesundheitskosten dazu zwingen, die günstigste Versor- gung für unsere Patienten zu wählen. Für den biologisch jüngeren Patienten scheint das Konzept der Erhaltung des Femurkopfes mit drei kanülierten Schrauben allgemein akzeptiert zu sein. Gute Knochenqualität und genügende Patientenkompliance bieten gute Voraussetzungen für eine ungestörte Heilung. Beim älteren Patienten über 65 Jahre braucht es neben einer anatomischen Reposition oder einer Valgusimpaktion eine gute Knochenqualität und einen hohen Aktivitätsgrad, keine relevanten Comorbiditäten, um gute Voraussetzungen für eine komplikationsfreie Osteosynthese zu gewährleisten. In der Altersgruppe der Patienten über 84 Jahre heilen $50 \%$ der dislozierten SHF nicht aus und erfordern Sekundäreingriffe [3]. Vor diesem Hintergrund macht sich zunehmend die Überzeugung breit, dass man in dieser Altersgruppe doch eher statt der Verschraubung eine prothetische Versorgung bei diesen Frakturen vornehmen sollte.

Auch wenn die Literatur keine Standardantwort auf die Frage nach der optimalen Behandlung der SHF geben kann, scheint in der Behandlung dieser häufigen Fraktur ein individualisiertes Vorgehen, unter Berücksichtigung der Faktoren Patientenalter, Aktivitätsgrad, Frakturtyp, Knochendichte und Comorbiditäten, gerechtfertigt. 


\section{Literatur}

${ }^{1}$ Asnis SE, Wanek-Sgaglione L. Intracapsular fractures of the femoral neck. Results of cannulated screw fixation. J Bone Joint Surg Am. 1994 Dec; 76 (12): 1793-803

${ }^{2}$ Baitner AC, Maurer SG, Hickey DG, Jazrawi LM, Kummer FJ, Jamal J, Goldman S, Koval $\mathrm{KJ}$. Vertical shear fractures of the femoral neck. A biomechanical study. Clin Orthop 1999 Oct; (367): 300-5

${ }^{3}$ Barnes R, Brown JT, Garden RS, Nicoll EA. Subcapital fractures of the femur. A prospective view. J Bone Joint Surg Br 1976; 58: 2 24

${ }^{4}$ Beck A, Ruter A. Femoral neck fractures diagnosis and therapeutic procedure. Unfallchirurg 1998 Aug; 101(8): 634-48

${ }^{5}$ Bonnaire F, Schaefer DJ, Kuner EH. Haemarthrosis and joint pressure in femoral neck fractures. Clin Orthop 1998; 353: 148-55

${ }^{6}$ Colles A. Fracture of the neck of the femur. Dublin Hosp Rep 1818; 2: 334-55

${ }^{7}$ Frandsen PA, Andersen E, Madsen F, Skjodt T. Garden's classification of femoral neck fractures. An assessment of inter-observer variation. J Bone Joint Surg Br 1988 Aug; 70(4): 588-90

${ }^{8}$ Fuglesang PN, Edna TH, Hatlinghus S, Svinsas $M$, Bjerkeset T. Femoral neck fractures treated with the hip compression screw. A follow-up study of 311 cases. Acta Orthop Scand Suppl 1992; 247: 6

${ }^{9}$ Gill TJ, Sledge JB, Ekkernkamp A, Ganz R. Intraoperative assessment of femoral head vascularity after femoral neck fracture. J Orthop Trauma 1998 Sep-Oct; 12(7): 474-8

${ }^{10}$ Goodman SB, Bauer TW, Carter D, Casteleyn PP, Goldstein SA, Kyle RF, Larsson S, Stankewich CJ, Swiontkowski MF, Tencer AF, Yetkinler DN, Poser RD. Norian SRS cement augmentation in hip fracture treatment. Laboratory and initial clinical results. Clin Orthop $1998 \mathrm{Mar}$ (348): 42-50

${ }^{11}$ Kauffman JI, Simon JA, Kummer FJ, Pearlman CJ, Zuckerman JD, Koval KJ. Internal fixation of femoral neck fractures with posterior comminution: a biomechanical study. J Orthop Trauma 1999 Mar-Apr; 13 (3): 155-9

${ }^{12}$ Leadbetter GW. A treatment for fracture of the neck of the femur. J Bone Joint Surg 1933; 15: $931-41$

${ }^{13}$ Lu-Yao GL, Keller RB, Littenberg B, Wennberg JE. Outcomes after displaced fractures of the femoral neck. A meta-analysis of one hundred and six published reports. J Bone Joint Surg Am 1994 Jan; 76 (1): 15-25

${ }^{14}$ Manninger J, Kazar G, Fekete G, Fekete K, Frenyo S, Gyarfas F, Salacz T, Varga A. Significance of urgent (within 6h) internal fixation in the management of fractures of the neck of the femur. Injury. 1989 Mar; 20(2): $101-5$
${ }^{15}$ Parker MJ, Blundell C. Choice of implant for internal fixation of femoral neck fractures. Meta-analysis of 25 randomised trials including 4,925 patients. Acta Orthop Scand 1998 Apr; 69 (2): 138-43

${ }^{16}$ Raaymakers ELFB, Schafroth M. Die mediale Schenkelhalsfraktur: Kontroversen in der Behandlung. Unfallchirurg 2002; 105: $178-86$

${ }^{17}$ Robinson CM, Saran D, Annan IH. Intracapsular hip fractures. Results of management adopting a treatment protocol. Clin Orthop 1994 May; (302): 83 -91

${ }^{18}$ Schmidt AH, Swiontkowski MF. Femoral neck fractures. Orthop Clin North Am 2002 Jan; 33 (1): 97 - 111

\section{Dr. med. Daniel Geissmann Oberarzt Chirurgie}

Priv.-Doz. Dr. med. Reto Babst Co-Chefarzt Chirurgie

Kantonsspital Luzern

Spitalstraße

CH-6000 Luzern 